\title{
Estimation of soil gas permeability for assessing radon risk using Rosetta pedotransfer function based on soil texture and water content
}

\author{
David Benaventea, ${ }^{a},{ }^{*}$ Javier Valdés-Abellán ${ }^{b}$, Concepción Plab, Enrique Sanz-Rubio ${ }^{c}$ \\ aDepartment of Earth and Environmental Sciences, University of Alicante, Alicante, Spain \\ ${ }^{b}$ Department of Civil Engineering, University of Alicante, Alicante, Spain \\ 'Geomnia Natural Resources SLNE, Madrid, Spain
}

* Corresponding author:

D. Benavente

Department of Earth and Environmental Sciences, University of Alicante, Alicante, Spain david.benavente@ua.es

Tef./FAX: +0034 965903737

Email list:

D. Benavente: david.benavente@ua.es

J. Valdés-Abellán: javier.valdes@ua.es

C. Pla: c.pla@ua.es

E. Sanz-Rubio: esanz@geomnia.es 


\section{ABSTRACT}

Radon is a natural source of radioactivity and it can be found in all soils and rocks in the Earth. The presence of radon gas in indoor environments implies a serious risk for human health, already listed as carcinogenic by the World Health Organization. The most relevant methods to infer the risk for radon exposure are based on soil radon concentration and gas permeability that describe the effective radon movement in the soil. However, they neglect crucial soil properties and water content in soil, which can affect greatly soil permeability to gases. Additionally, soil permeability measurement remains expensive, difficult and time-consuming. In this paper we show a new and simple methodology to infer radon risk based on Rosetta3 pedotransfer function as well as soil texture and water content. We also determine the influence of soil texture both on the gas permeability variation in dependence on water content and on the parameter $n$ of the van Genuchten -Mualem model, which establishes the shape of the relative permeability curves. We show that radon risk exposure may change importantly for the same soil with different soil water contents. We finally apply and validate the proposed method using radon permeability data from the Canadian component of the North American Soil Geochemical Landscapes Project (NASGLP). Results highlight that the proposed methodology provides reliable estimations of the gas permeability and reveal that the presence of water content may cross the boundary between two radon risk categories, and consequently, may change the radon risk category to safer situations.

\section{KEYWORDS}

Radon risk, soil properties, gas permeability, Rosetta, soil water content 


\section{INTRODUCTION}

The isotope ${ }^{222} \mathrm{Rn}$ of radon is a natural source of radioactivity, participating in the disintegration chain of uranium, ${ }^{238} \mathrm{U}$, and present in all soils and rocks in the Earth surface. Radon gas is not only a natural tracer for geological processes (Barbosa et al., 2015) but also a clear risk for human health when released to indoor environments. The World Health Organization, WHO, has highlighted the existence of a doubtless correlation between long exposure to high radon concentration and lung cancer (W. H. O., 2009), and the US Environmental Protection Agency, US-EPA, considers radon as the second leading cause of lung cancer, being responsible for 21,000 deaths per year in USA (https://www.epa.gov/radon/health-risk-radon\#iow). Moreover, radon is suspected to cause other types of cancer such as skin cancer (López-Abente et al., 2018, Vienneau et al., 2017). ${ }^{222} \mathrm{Rn}$ disintegration produces $\alpha$ particle and other radionuclides, which may be inhaled by humans and be an important source of radiation. This risk increases in areas with high natural ${ }^{222} \mathrm{Rn}$ concentration in soils and/or soils with high gas permeability (Font, 2009). Jones (1999) already showed that indoor ${ }^{222} \mathrm{Rn}$ concentration could impose a serious health risk and more recently Stauber et al., (2017) monitored 222Rn concentration in residential houses in USA reporting that radon concentration of $18 \%$ implied moderate risk (above $74 \mathrm{~Bq} / \mathrm{m}^{3}, 2 \mathrm{pCi} / \mathrm{L}$, according to $\mathrm{EPA}$ ) for human health and high risk (above $148 \mathrm{~Bq} / \mathrm{m}^{3}, 4 \mathrm{pCi} / \mathrm{L}$, according to EPA) for $4 \%$ of the houses.

Several attempts have appeared in the last decades aiming to identify risk areas at different scales. Thus, the US-EPA elaborated a map in 1993 (US Environmental Protection Agency, 2003), later updated, which classifies the US territory into three levels according to the natural presence of radon in soil. Remarkably, the Czech Republic government developed a method to identify risk areas, which is based on radon gas concentration and soil permeability and can be considered as the most common method (Neznal et al., 2004). Finally, national health radon programs, such as those in Norway (Watson et al., 2017) and Ireland (Elío et al., 2018), assessed indoor radon concentration based on regional geology. Among all the methods that can be found in scientific literature, government reports and/or normatives, the risk levels (normally three levels) of radon exposure are based only on ${ }^{222} \mathrm{Rn}$ concentration, or at best on ${ }^{222} \mathrm{Rn}$ concentration jointly with gas permeability of soil at field conditions.

Radon emanation and transport in soils determine strongly the effective entrance of radon into the buildings. Radon concentration in soils is linked to radon emanation and is described by the emanation coefficient, also termed as emanation fraction or emanating power. It is defined as the fraction of radon atoms generated that escape the solid phase in which they are formed and become free to migrate through the bulk medium. The emanation power depends on the grain size, water content and temperature of the material (Zhuo et al., 2006; IAEA TRS 474, 2013). The particle size and shape determine in part how much uranium and radium is close enough to the surface of the grain to allow the radon to escape into the interstitial pores. Thus, the emanation coefficient increases as 
grain size decreases, if radium is uniformly distributed throughout a grain. Water content enhances the emanation coefficient because water increases the direct recoil fraction of emanation. Finally, the temperature can be considered of minor importance over the range of temperatures common for surface soils (Nazaroff, 1992).

Gas permeability is mainly considered because advection is generally more important than diffusion for radon transport into buildings. Soils are three-phase complex mediums where the voids are occupied by water or gas intermittently and soil properties such as texture, bulk density, organic matter, etc., or soil water content directly affect gas permeability. Soil gas permeability is inversely correlated to soil water permeability, and both are related to soil water content. Additionally the pore size distribution also affects the relation between both permeabilities and soil water content (Van Looy et al., 2017). Neglecting these facts just leads to non-accurate estimations of risk associated to radon. Therefore, an accurate description and characterisation of soil properties is essential. However, this soil characterisation, mainly soil permeability, continues to be expensive, difficult and time-consuming at present time (Zhang et al., 2017). Scanlon et al. (2001) reported that the comparison of gas permeabilities from laboratory and field indicates that field derived estimates of gas permeabilities generally exceed laboratory derived estimates up to some orders of magnitude (Weeks, 1978; Edwards, 1994). These differences in permeability are attributed primarily to the increase in scale from laboratory to field measurements and inclusion of macropores, fractures and heterogeneities in field measurements. Field permeability measurements of low permeability media include the effects of viscous slip and Knudsen diffusion.

In order to solve, or at least ameliorate this situation, during last decades pedotransfer functions (PTFs) have served as an alternative and less-expensive way to determine soil hydraulic properties. PTFs have a long history in soil science and eager readers can find a detailed description in papers such as Zhang and Schaap (2017) or recent and very good reviews such as Van Looy et al., (2017). Each PTF was obtained for specific datasets and normally limited to specific areas. Among them, Rosetta is one of the most extended among Soil Science. It determines the soil hydraulic parameters according to the van Genuchten-Mualem constitutive relationships (Mualem, 1976, van Genuchten, 1980) and it is implemented in soil water flux and transport models such as Hydrus1D (Šimůnek et al., 2013). Very recently, Zhang and Schaap (2017) published a new version of Rosetta, called Rosetta3, solving several deficiencies, like the impossibility to provide covariance matrix between soil water retention properties and saturated water permeability and introducing a new set of hierarchical functions. With such a PTF, soil hydraulic (and complementarily soil gas) properties can be estimated from standard soil properties such as the percentages of sand, silt and clay and the bulk density.

The main objective of this study is the estimation of soil gas permeability for radon risk studies using Rosetta pedotransfer function. For this proposal, we determine the influence of the texture, bulk density and water content of soils on the unsaturated gas 
permeability and the parameter $n$ of the van Genuchten -Mualem model in order to recommend the most efficient and quickest methodology for quantifying the effective transport of radon in soils. To validate the methodology, results are compared with the database of the Canadian component of NASGLP, performed for the assessment of radon potential risk in Health Canada's National Radon Program. Furthermore, these results are tested with the radon potential risk of building sites of the Czech Republic, which combines both the radon concentration in soil and soil gas permeability. In addition, we also evaluate the variations in the classifications of the potential radon risk in field situations due to the presence of soil water and their modifications by future changes in the climate conditions. The practical findings of this paper will allow a more accurate diagnosis of radon risk for human health, which can be implemented in national, regional or local health radon programs.

\subsection{General equations for gas permeability in soils.}

Risk assessment of exposure to radon and other volatile contaminants in buildings and confined space requires study of the gas transport mechanism. However, in the literature, transport mechanisms of the liquid phase are more extended than of the gas phase. For this reason, in this section, we will describe the gas transport equations and relate them to water content. An interesting and detailed review of the soil gas movement in unsaturated systems can be found in Scanlon et al. (2001).

As we commented in the previous section, advection is generally considered more important than diffusion for radon transport into buildings. Meteorological parameters, such as precipitation, wind speed and direction, temperature, relative humidity and barometric pressure can affect the advective flux (IAEA TRS 474, 2013). Advective gas transport is described by Darcy's law and varies with permeability and pressure gradient. For a single-phase gas flow, intrinsic gas permeability or gas permeability, $\mathrm{k}_{\mathrm{g}}\left(\mathrm{L}^{2}\right)$, has area units and only depends on porous media medium. Gas conductivity, $\mathrm{K}_{\mathrm{g}}\left(\mathrm{L}^{-\mathrm{T}^{-1}}\right.$ ), (and hydraulic conductivity for liquid water) has units of velocity and considers both porous media and fluid properties (gas density, $\rho$, and gas viscosity, $\mu$ ). For any fluid,

$$
K=k \cdot(g \cdot \rho / \mu)
$$

where $g$ is the gravitational acceleration constant. According to Darcy's law, intrinsic permeability is independent of the type of fluid and, therefore, intrinsic permeabilities for gas and liquid water are equal.

In the unsaturated zone, the soil pore space generally contains gas and liquid. Darcy's law is applied to each fluid in the system, which assumes that there is no interaction between the fluids (Dullien, 1979). The presence of both fluids decreases the cross-sectional area available for the flow of each fluid and its permeability with respect to that fluid decreases (Scanlon et al., 2001). Consequently, the flow of that fluid also decreases. Depending on the degree of soil water saturation, liquid water can cover the 
pore surface, can form static menisci and/or can fill the interparticle pore space. Thus, the presence of water in the porous materials reduces both connected porosity and pore size and increases the tortuosity (Benavente and Pla, 2018). In other words, gas permeability decreases as water content increases.

For a given gas saturation, $S_{g}$, unsaturated gas permeability, $\mathrm{k}_{\mathrm{g}}\left(\mathrm{S}_{\mathrm{g}}\right)$, ranges from a maximum considering a situation $100 \%$ gas saturated $\left(0 \%\right.$ saturated in liquid water, $S_{w}$ ) to zero for saturated water condition. If we consider that pore space only contains gas and liquid, then $S_{g}+S_{w}=1$. The relative permeability of the gas, $k_{r}, g$, is a function of the gas saturation, $S_{g}$, and it is defined as the permeability of the unsaturated medium at a particular gas saturation, $\mathrm{kg}_{\mathrm{g}}$. In other words, $\mathrm{k}_{\mathrm{r}, \mathrm{g}}=\mathrm{kg}\left(\mathrm{S}_{\mathrm{g}}\right) / \mathrm{kg}_{\mathrm{g}}$. In the case of liquid water, $\mathrm{k}_{\mathrm{r}, \mathrm{w}}=\mathrm{k}_{\mathrm{w}}\left(\mathrm{S}_{\mathrm{w}}\right) / \mathrm{k}_{\mathrm{w}}$.

We consider the soil hydraulic parameters as described by the van GenuchtenMualem (VGM) model (Mualem, 1976, van Genuchten, 1980), for liquid and gas as follow:

$$
\begin{aligned}
& S_{w}=\frac{\theta(h)-\theta_{r}}{\theta_{S}-\theta_{r}}=\frac{1}{\left(1+(\alpha h)^{n}\right)^{m}} \\
& k_{r, w}=\frac{k_{w}\left(S_{w}\right)}{k_{w}}=S_{w}^{\tau}\left[1-\left(1-S_{w}^{m^{-1}}\right)^{m}\right]^{2} \\
& k_{r, g}=\frac{k_{g}\left(S_{w}\right)}{k_{g}}=\left(1-S_{w}^{\tau}\right)\left[1-\left(1-\left(1-S_{w}\right)^{m^{-1}}\right)^{m}\right]^{2}
\end{aligned}
$$

where $h$ is the soil pressure head $(\mathrm{cm}) ; \theta, \theta_{r}$ and $\theta_{s}$ are the actual, residual and saturated volumetric water contents respectively $\left(\mathrm{cm}^{3 \cdot} \mathrm{cm}^{-3}\right) ; \alpha\left(\mathrm{cm}^{-1}\right), n(-)$, and $\tau(-)$ are empirical coefficients that determine the shape of the hydraulic functions. $\alpha$ is related to the air entry value, $n$ is related to the pore size distribution, and $\tau$ is the tortuosity parameter normally assumed equal to $0.5 . m$ (dimensionless) is an empirical parameter calculated by $m=1-n^{-1}$. Both unsaturated water and gas permeabilities are expressed as a function of the effective water saturation, $S_{w}$.

As we previously commented, Darcy's law theoretically considers that intrinsic permeabilities for gas and liquid water are equal. However, from low to moderate water saturation, gas preferably fills and flows in larger pores and consequently, the relative permeability of the gas phase is greater than that of the liquid phase (Demond and Roberts, 1987). Moreover, Scanlon et al. (2001) argued that gas slippage (Klinkenberg effect) causes higher gas permeability than water permeabilities in fine-grained media or under small mean pressures. Gas slippage is negligible in medium-coarse media having high mean pressures. On the contrary, air conductivities are about one-tenth of hydraulic conductivities because the air density is approximately three orders of magnitude less than water density and air viscosity is approximately 50 times less than water viscosity. These discrepancies can occur for coarse media and can be corrected for the calculation of gas permeability using Eq. (1). Furthermore, the fluid transports close to gas and water saturation behave differently. Thus, the rate of relative gas permeability change at low $S_{w}$ values (high values of $S_{g}$ ) is lower than equivalent water relative permeability change at 
high $S_{w}$ values (low values of $S_{g}$ ). In short, relative permeabilities do not sum to one (Scanlon et al., 2001). Moreover, although $\mathrm{k}_{\mathrm{r}, \mathrm{g}}$ is close to one, it presents a hysteretic behaviour and depends on saturation history (i.e., whether the system is drying or wetting). At a given water saturation, gas permeability is generally greater for wetting than for drying (Stonestrom and Rubin, 1989). Consequently, field measurements in shortterm studies of radon risk can yield uncertain results because it represents only a snapshot of a highly dynamic system, with a strong dependence on soil saturation content and history.

Transferring these insights to our research subject reveals that radon movement will enhance for low water content, which is mainly produced by drying soil and it will tend to zero as water content increases because connected porosity and pore size decrease and cause a reduction in radon permeability.

Figure 1 displays the variation of gas and water relative permeabilities, $\mathrm{k}_{\mathrm{r}, \mathrm{g}}$ and $\mathrm{k}_{\mathrm{r}, \mathrm{w}}$ respectively, versus the effective water saturation, $S_{w}$. The shape of the relative permeabilities is sensitive to the pore size distribution and therefore to the soil texture. The parameter $n$ of the van Genuchten-Mualem model is related to pore size and presents smaller values for fine soils. Thus, sandy soils present the highest $n$ values $(n=4.47$ for $100 \%$ sand), silt soils intermediate ( $n=1.67$ for $100 \%$ silt) and the lowest values for clayey soils ( $n=1.18$ for $100 \%$ clay).

On the other hand, $n$ influences the shape of the relative permeability curves. Soil textures with small pore sizes and lower $n$ values displays an abrupt shape, thus the effective fluid transport is produced close to saturated conditions. In other words, radon flux is effective for low to very low $S_{w}$ values for clayey soils whereas it occurs from medium to very low $S_{w}$ values in sandy soils (Fig. 1).

\section{MATERIALS AND METHODS}

\subsection{Soil texture and hydraulic properties.}

In the present study, we adopted the PTF Rosetta3 (Zhang et al., 2017) to estimate the unsaturated hydraulic properties of the soil. PTFs relate simple-to-measure soil properties to less available parameters of Earth system processes and their importance in Earth System Science has been recently highlighted in Van Looy et al., (2017). Among the available PTFs in the literature, we chose Rosetta as it constitutes a mainstream in Soil Science. Former versions of Rosetta (Rosetta1) are even implemented in well-extended software of soil water/solute/gas movement such as Hydrus1D (Šimůnek et al., 2013).

Rosetta determines the soil hydraulic parameters according to the van GenuchtenMualem constitutive relationships (Mualem, 1976, van Genuchten, 1980). Rosetta offers five different PTFs that allow the prediction from different levels of information according to available data: (i) soil textural classes sand; (ii) silt and clay percentages (SSC); (iii) SSC

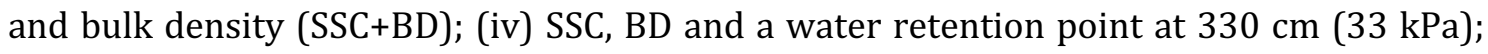


and (v) SSC, BD and water retention points at 330 and $15000 \mathrm{~cm}$ (33 and $1500 \mathrm{kPa})$. All estimated hydraulic parameters are accompanied by uncertainty estimates that permit an assessment of the reliability of Rosetta's predictions (see Schaap and Leij (1998) and Schaap et al. (2001) for more information). Among those PTFs, we evaluated only two options: the SSC and the SSC+BD in order to maintain the interest and attractiveness of the proposed methodology. More elaborated options of inputs using Rosseta require the determination of volumetric water content at one or more soil pressure head values, which increases the time invested and the devices needed to get such information. Between those options, according to Schaap et al. (2001) and Zhang and Schaap (2017), the SSC+BD option is generally more accurate than the SSC. Moreover, for very clayey soils, the estimations tend to have more uncertainty (variability, larger standard deviations) because Rosetta's calibration database had only very few clayey samples.

All potential combinations of sand, silt and clay with increments of $1 \%$ were considered in the study leading a total number of 5151 combinations. Considered bulk density values included in this study range from 1.25 to $1.80 \mathrm{~g} \cdot \mathrm{cm}^{-3}$ including the most common values for non-organic soils and more than $85 \%$ of all soils in UNSODA database (Nemes et al., 2001). We evaluate the influence of bulk density in the Rosetta3 predictions of $k_{g}$ and $n$ to determine the usefulness of its determination that requires a specific field sampling and lab characterisation.

In this paper, we use USDA-based soil texture classes for radon risk studies because (i) it is widely used and established in soil science studies; (ii) these studies are frequently linked to the estimation of unsaturated hydraulic properties; and (iii) textural characteristics are among the most easily measurable soil properties. Additionally, Rosetta considers the USDA classification system, where size boundaries are set at $2 \mu \mathrm{m}$ for claysilt, at $50 \mu \mathrm{m}$ for silt-sand, and at $2 \mathrm{~mm}$ for sand-gravel. USDA classification system presents 12 soil texture classes as a function of sand, silt, and clay percentages (SSC) in a ternary diagram, which was proposed by Davis and Bennett (1927). In the literature, there are several texture-based classifications, such as ISSS (International Soil Science Society) and USDA (U.S. Department of Agriculture), although the latter classification system is perhaps the most widely recognized.

Finally, since Rosetta1 is still working and is implemented in commercial software, a comparison between the Rosetta1 and Rosetta3 is needed, which has been included in the Supplementary Material section.

\subsection{Calculation of gas permeability from soil hydraulic conductivity.}

We aim to calculate gas permeability values and their variations with water content for different soils. These results will allow us to predict the potential risk of radon exposure. 
The calculation of gas permeability requires the characterisation of sand, silt and clay percentages and, optionally, the bulk density values using standard soil tests. Thus, the proposed methodology for the calculation of gas permeability for given water content is as follows:

a) Soil properties: characterisation of sand, silt and clay percentages and, optionally, the bulk density using standard soil tests.

b) Rosetta predictions: we obtain the hydraulic conductivity and water retention parameters for each specific soil. From all Rosetta predictions we only need parameter $n$ and hydraulic conductivity, $\mathrm{K}_{\mathrm{w}}$, to obtain the gas permeability.

c) Calculation of gas permeability: we firstly convert hydraulic conductivity, $\mathrm{K}_{\mathrm{w}}$ $\left(\mathrm{cm} \cdot\right.$ day $\left.^{-1}\right)$, to intrinsic permeability, $\mathrm{k}_{\mathrm{w}}\left(\mathrm{m}^{2}\right)$, using Eq. (1). Thus, for pure water at $20^{\circ} \mathrm{C}, \mathrm{k}_{\mathrm{w}}$ $\left(\mathrm{m}^{2}\right)=1.18 \cdot 10^{-14} \cdot \mathrm{K}_{\mathrm{w}}\left(\mathrm{cm} \cdot \mathrm{day}^{-1}\right)$. Secondly, as we previously argued, we consider that intrinsic permeabilities for gas and liquid water are equal according to Darcy's law. That is: $\mathrm{k}_{\mathrm{g}}\left(\mathrm{m}^{2}\right)=\mathrm{k}_{\mathrm{w}}\left(\mathrm{m}^{2}\right)$. This value considers the $100 \%$ gas saturated $(0 \%$ saturated in liquid water, $S_{w}$ ) and corresponds to the maximum permeability value of given soil that permits the radon flux. Although the working hypothesis of $\mathrm{k}_{\mathrm{g}}=\mathrm{k}_{\mathrm{w}}$ is based on the theoretical Darcy's law, some discrepancies might be found between $k_{g}$ and $k_{w}$ as was highlighted in the previous section. Consequently, the $\mathrm{k}_{\mathrm{g}}=\mathrm{k}_{\mathrm{w}}$ assumption should be considered in each specific research.

d) Influence of water content: the water content, $S_{w}$, and parameter $n$ are introduced in Eq. (4) and we obtain the relative permeability to the gas, $\mathrm{k}_{\mathrm{r}, \mathrm{g}}$. The unsaturated gas permeability for partially saturated soil, $\mathrm{k}_{\mathrm{g}}\left(\mathrm{S}_{\mathrm{w}}\right)$, can be calculated as $\mathrm{k}_{\mathrm{g}}\left(\mathrm{S}_{\mathrm{w}}\right)=\mathrm{k}_{\mathrm{r}, \mathrm{g}} \cdot \mathrm{kg}_{\mathrm{g}}$. We calculate the gas permeability as a function of the effective water saturation rather than gas saturation because $S_{w}$ is the most extended form for expressing the gas-water saturation degree and it is the parameter obtained in the lab. The unsaturated gas permeability of the soil quantifies the real radon flux in a natural system and reflects the reduction of radon movement by the presence of water in the soil. We can obtain the natural water content of the sampled soil using standard soil tests or can compute different water contents using Eq. (4).

For simplicity and analogy to other pKs expressions in science (ej.: $\mathrm{pH}, \mathrm{pK}$, pe, etc.), we defined $\mathrm{pK}_{\mathrm{g}}$ as:

$$
p K_{g}=-\log \left(k_{g}\right)
$$

Thus, low $\mathrm{pK}_{\mathrm{g}}$ values means permeable soils for gas movement whereas impermeable soils present highest $\mathrm{pK}_{\mathrm{g}}$ values. 


\subsection{Validation of methodology using extensive review of radon studies.}

In order to validate the proposed methodology, we use the database of the Canadian component of the North American Soil Geochemical Landscapes Project (NASGLP) carried out in 2004 (Friske et al., 2014). A significant part of the Canadian component of the NASGLP included the collection of soil texture, soil gas radon, permeability and gammaray spectrometric analysis. These data and soil geochemical data from the NASGLP project were addressed to assess the radon potential risk included in Health Canada's National Radon Program.

Radon concentration and permeability of soils were obtained in the field at $60 \mathrm{~cm}$ depth. Soil gas samples were collected and analysed using an RM-2 Radon detector. Gas permeability was measured using the RADON-JOK (C) equipment for in situ permeability measurements. Soil texture, density and natural water content were obtained in the lab. The procedure for grain size analysis includes sand, silt and clay determination and complete grain size analysis. The classes of sizes greater than $0.063 \mathrm{~mm}$ were determined by wet sieving through a stack of appropriately sized sieves, whereas the classes of sizes less than $0.063 \mathrm{~mm}$ were determined using a particle size analyzer. The sand-silt limit stablished in the NASGLP project is higher than the USDA limit of $50 \mu \mathrm{m}$ which is the standard adopted in Rosseta pedotransfer function. This slightly overestimates the silt fraction and underestimates the sand percentages in the NASGLP data, which may affect the results of the validation. Soil bulk density, $\rho_{b}$, was defined as the ratio of the mass of an oven-dry soil sample to the volume of that sample. Water content, $\theta_{\mathrm{wg}}$, was determined gravimetrically as the weight of water in a given mass of soil to the weight of the dried sample expressed as a percentage. The volumetric water content was calculated as $\theta_{\mathrm{wg}} \cdot \rho_{\mathrm{b}} / \rho_{\mathrm{w}}$, where $\rho_{\mathrm{w}}$ is the density of liquid water (for a detailed description of the field and lab measurements, see Friske et al., 2014).

From the database, we selected the sample sites that satisfy the following requirements: (i) reproducible values of gas permeability (avoiding measurements with no gas movement seen after first 5 minutes as well as estimated values, respectively); and (ii) soil radon values higher than $0.7 \mathrm{kBq} \mathrm{m}^{-3}$ (considered as background radon concentration) and reproducible values (coefficient of variation lower than 50\%). The obtained values are mainly classified as sandy loam to silty loam categories and, unfortunately, there are no data points in the clayey soil domain.

\section{RESULTS AND DISCUSSION}

\subsection{Estimated soil properties. Influence of water content on unsaturated gas permeability.}

Soil properties affect the shape parameter of the van Genuchten-Mualem model $n$ and permeability. Figure 2 collects the Rosseta3 estimations for those two parameters 
using SSC as the input information and using SSC+BD, with bulk density ranging from 1.25 to $1.80 \mathrm{~g} \cdot \mathrm{cm}^{-3}$ for sandy, silty and clayey soils. Estimations with the simplest option (SCC) report $n$ and $\mathrm{pK}_{\mathrm{g}}$ estimations similar to those obtained with the option (SSC+BD) using bulk density values that include the majority of soils from the UNSODA database. Accordingly, we conclude that the SSC option would increase simplicity of the proposed methodology without reducing accuracy significantly, making the methodology simpler. Hence, we adopted this alternative as the unique input parameter to obtain the soil properties with Rosetta3.

Figures 3 and 4 highlight the strong influence of water content on gas permeability. Figure 3 depicts $\mathrm{pK}_{\mathrm{g}}$ values in a soil texture triangle USDA diagram for different water content. As example, Figure 3a displays the loam, sand, silt, and clay categories in the USDA diagram. Gas permeability drops several orders of magnitude with the presence of water, becoming significant for $S_{w}$ higher than 0.5 as Figure 1 describes. Thus, liquid water in soil pore space reduces gas transport, which gradually makes soils more impermeable to gas (Figure 4). Figure 1 showed that $n$ influences the shape of the relative permeability curves. Thus, clayey soils with lower $n$ values display a steep slope for $S_{w}<0.05$, and therefore, a higher gas permeability reduction rate.

Bulk density affects the soil microstructure, although its influence on $\mathrm{pK}_{\mathrm{g}}$ and $n$ values is of minor order (Fig. 2). For a given soil, if the bulk density increases, soil porosity and pore size decrease. As a consequence, soil becomes less permeable (high values of $\mathrm{pK}_{\mathrm{g}}$, Fig. 2) and the parameter $n$ of the van Genuchten-Mualem model reaches lower values by the pore size reduction (Fig. 2).

In the Supplementary material, Figures S1 and S2 and Tables S1 and S2 compare the estimations $\mathrm{pK}_{\mathrm{g}}$ and $n$ using Rosetta1 and Rosetta3. The influence of bulk density on these parameters is also neglectable. However, some differences are found. $\mathrm{pK}_{\mathrm{g}}$ and $n$ values

obtained using Rosetta1 are slightly higher than in Rosetta3, although their differences are below $5 \%$.

These results present interesting practical conclusions for the estimation of gas permeability from the proposed methodology: the measurement of bulk density is not mandatory (although recommended) and the election of the Rosetta version is not crutial.

\subsection{Validation study.}

The proposed methodology is compared with the database of the Canadian component of NASGLP (Friske et al., 2014). The selected values from the data set are mainly classified as sandy loam to silty loam categories (the lower part of the soil texture triangle), with no data points in the clayey-dominated classes.

Figure 5 compares field gas permeability and unsaturated gas permeability using the water content obtained in the field, $\mathrm{S}_{\mathrm{w}}$. Results reveal that the proposed methodology 
provides reliable estimations of the gas permeability $\left(R^{2}=0.685\right)$. In general, predicted unsaturated gas permeabilities are slightly lower (higher values of $\mathrm{pK}$ ) than field values, mostly less than one order of magnitude (pK scale is logarithmic).

This discrepancy may be related to both the proposed methodology and the experimental values. On the one hand, the proposed methodology calculates hydraulic conductivity (and $n$ ) from Rosetta3 PTF and assumes that $\mathrm{k}_{\mathrm{w}}=\mathrm{k}_{\mathrm{g}}$. In other words, the calculated intrinsic and unsaturated gas permeabilities are obtained from water transport properties instead of gas measurements. As we previously mention, from low to moderate water saturation or fine-grained media or under small mean pressures, the relative permeability of the gas phase is greater than that of the liquid phase (Scanlon et al. 2001). Consequently, field gas permeabilities will show higher values of permeability (lower values of $\mathrm{pK}$ ) than calculated unsaturated gas permeabilities, which are computed from water transport coefficients.

On the other hand, experimental gas permeability measurements present difficulties and discrepancies. As we will report later, the modification of the soil structure due to the installation of measurement devices and the presence of macropores and fractures in the soil, for instance, may create a preferential flow that increases gas movement. As a result, the field measurements will overestimate field gas permeabilities, which also provide higher values of gas permeability than the calculated unsaturated gas permeabilities.

The presence of water remarkably decreases the radon flux through the soil, and consequently, modifies the effective movement of radon into the building. Figure 6 represents the radon potential risk of building sites proposed by Neznal et al., (1995) and combines both the radon concentration in soil and soil gas permeability. It highlights the important reduction of gas permeability for different soil textures and water content below $S_{w}=0.4$, where gas transport is more effective. For high values of $S_{w}$, gas transport will be drastically hindered as we previously reported. Thus for a given radon concentration, the presence of water content may cross the boundary between two radon risk categories. Consequently, the presence of liquid water in the soil may change the radon risk category towards lower risk categories. These findings also reveal that soil moisture conditions highly affect the observed permeability and consequently may affect the radon risk classification. This dependence highlights the necessity of correction of permeability measurements (and radon gas measurements as well) to achieve comparability.

These modifications in gas permeability are important even for small variations in the water content and they are related to soil texture. Thus, for example, radon flux is effective from very low to low $S_{w}$ values for clayey soils and strongly reduced for medium to high $S_{w}$, whereas in sandy soils the radon transport occurs from very low to medium $S_{w}$ values.

Figure $6 \mathrm{~b}$ plots field data from the NASGLP and estimations using the presented methodology for the radon potential risk classification. Estimated values include the 
(intrinsic) gas permeability, $\mathrm{k}_{\mathrm{g}}$, and the unsaturated gas permeability, $\mathrm{k}_{\mathrm{g}}\left(\mathrm{S}_{\mathrm{w}}\right)$, using the water content obtained in the field, $S_{w}$. In general, the estimations of unsaturated gas permeabilities present lower values of permeability (higher values of $\mathrm{pK}$ ) than the experimental data set. As we previously argued, this discrepancy may be related to the proposed methodology and the experimental values and also may be attributed to the strong influence of water content on the calculation of the unsaturated gas permeability.

We conclude that the proposed methodology provides reliable gas permeability values and two permeability parameters $\left(\mathrm{k}_{\mathrm{g}}\right.$ and $\mathrm{k}_{\mathrm{g}}\left(\mathrm{S}_{\mathrm{w}}\right)$ ) essential for radon studies only from soil texture. On the one hand, intrinsic gas permeability represents the maximum values of gas permeability that radon can flow and determines the soil radon permeability that produces major potential risk. On the other hand, unsaturated gas permeability that considers water content describes the effective radon movement under field conditions.

\subsection{Potential applications of the proposed methodology.}

The new methodology presents several potential applications that may enhance the estimation of the radon potential at different temporal and spatial scales.

The elaboration of a radon risk map may include different types of input data as indoor radon data, building architecture, climate, and the geogenic radon potential (Neznal et al., 2004; Szabó et al., 2014, Pásztor et al., 2016; Elío et al., 2017; ). Geogenic radon potential (GRP) provides information about the source of indoor radon concentration and considers that the major source of indoor radon concentration is the soil gas radon (Szabó et al., 2014). There are different methodologies to define the GRP of an specific region, which are based on several measured parameters such as numerical (permeability, ${ }^{222} \mathrm{Rn}$ concentration in soil air, and ${ }^{226} \mathrm{Ra}$ concentration) or geological data as categorical controls (Bossew et al., 2014). One of the most recognized methods for the GRP calculation of an area was established by Neznal et al. (2004). It considers field measurements of the radon concentration in soil gas and the gas permeability of soils. Generally, GPR calculation in the latter method is determined by a single measurement from one season, although the risk for human health due to radon arises from long-term exposure to certain doses. Thus, field measurements could be normalized to a reference soil water content or to the site-specific mean soil water content, which includes the expected variations of mean soil water content throughout the study area. In addition, the proposed methodology could be used for permeability estimations in GRP large-scale mapping, if a representative water content and soil texture data are available.

Another potential application of the new methodology can be inferred after the analysis of figure 6 that highlights that small changes in water content can greatly modify radon flux and can even modify the radon risk category. As a consequence, the impact on the radon risk under the different climate scenarios predicted by the IPCC in its last report (IPCC, 2013) should be worth studied, especially in regions where drier conditions are expected because drier soils will present higher gas permeabilities (and then higher radon 
risk classification) because soil pore space is gas saturated. Moreover, in clayey soils, the desiccation of clays will yield cracking and development of preferred pathways for gas transport (Jury and Horton, 2004). On the contrary, water saturated soils will reduce the available pore space of gas transport and will have lower gas permeability (lower radon risk classification). If water movement is significant the radon transport dissolved in water should not be disregarded.

Additionally the proposed methodology provides a tool to define strategies in housing design in order to reduce radon risk in locations with high natural concentrations. Soil water content, and consequently radon risk, can strongly vary due to changes in local water table, irrigation, evapotranspiration, rain events, drought, or even due to modification in buildings or ventilation systems.

Finally, radon emanation and gas permeability contribute to effective entrance of radon into the buildings, although they are oppositely related to grain size and water content. For example, gas permeably is higher for sandy (large grain size) and dry soils, whereas emanation coefficient has higher values for clayed (small grain size) and water saturated soils. The main objectives of the proposed methodology do not deal with radon emanation. However, the proposed model may be improved considering the changes of the emanation coefficients, E, using empirical emanation model available in literature (Zhou et al., 2006; IAEA TRS 474, 2013) as follow:

$$
\mathrm{E}=\mathrm{E}_{0}\left[1+\mathrm{k}_{1}-\exp \left(-\mathrm{k}_{2} \mathrm{~S}\right)\right] \text {, }
$$

where $E_{0}$ is the radon emanation coefficient of oven-dried soil, $S$ is the fraction of soil water saturation, and $\mathrm{k}_{1}$ and $\mathrm{k}_{2}$ are the regression coefficients of Eq. (6) for different soil textures. Zhou et al. (2006) reported $\mathrm{k}_{1}$ and $\mathrm{k}_{2}$ values for clay, silt and sand soils: $\mathrm{k}_{1}$ is equal to $1.85,1.73$ and 1.53 and $\mathrm{k}_{2}$ is $18.8,20.5$ and 21.8 for clay, silt and sand soils respectively.

However, the proposed methodology for the estimation of gas permeability itself and the experimental characterisation of soil permeability presents some limitations that should be considered for radon risk studies:

i) Rosetta. Twarakavi et al. (2009) argued that the soils in the database were not distributed evenly along all textures, being more robust for soils belonging to the sandand loam-dominated textures. Thus, the Rosetta training database works accurately for these soil textures.

ii) Validation data sets. A major issue in testing our research was the lack of available information for developing a comprehensive soil dataset that includes radon concentration, hydraulic properties, bulk density and the relative proportions of sand, silt, and clay contents that cover a wide range of textural combinations. The methodology was tested using soils mainly from sandy loam to silty loam categories and without data points in the clayey-dominated classes. An extended validation should be addressed considering soils from the clayey soil domains. 
iii) Gas permeability measurements. Gas permeability measurements in both laboratory and field present difficulties and discrepancies. These differences may correspond to the modification of the soil structure due to installation of measurement devices; the presence of organic matter, and macropores, fractures and other noncapillary pores in the soil such as desiccation cracks in clays, wormholes and root tubules. Scanlon et al. (2001) reported that a preferential flow can occur in this macropores because they drain rapidly and they are generally dry. Thus, the preferential flow of gases should therefore be much greater than that of liquids. The scale effect and domain heterogeneity (in terms of soil physical characteristics and water content) may also create considerable variations in the measured gas transport coefficients (Mohammadi and Vanclooster, 2019; Pla et al., 2017).

\section{CONCLUSIONS}

In this paper, we proposed a new methodology that estimates gas permeability based on soil texture and water content for radon studies. It is based on the well-established Rosetta pedotransfer function and provides a fast and accurate estimation of intrinsic gas permeability and the parameter $n$ of the van Genuchten-Mualem model for unsaturated soils. The analysis of the results from the proposed methodology confirms that the measurement of bulk density is not mandatory (although recommended) and that both Rosetta $1 \& 3$ provide similar results.

The presence of water remarkably decreases the radon flux through the soil, and consequently, modifies the effective movement of radon into dwellings. Gas permeability variation with water content is sensitive to soil texture. Thus, small particle size causes lower gas permeability values and the parameter $n$ displays more abrupt shapes.

In terms of radon risk studies, the proposed methodology provides two relevant gas permeability values. On the one hand, intrinsic gas permeability represents the upper limit of gas permeability (maximum radon flow). It determines the soil radon permeability that produces a major potential risk, although it could lead to inaccurate classifications of the potential radon risk in real situations, since it assumes absence of water. On the other hand, unsaturated gas permeability that considers water content describes the effective radon transport under field conditions.

The proposed methodology is compared with the database of the Canadian component of NASGLP and evaluated using the radon potential risk of building sites proposed by Neznal et al., (1995), which combines both the radon concentration in soil and soil gas permeability. Although the selected values from the data set are mainly classified as sandy loam to silty loam categories, the estimations of gas permeabilities are promising. This comparison reveals that calculated unsaturated gas permeabilities are slightly lower than field values with differences of less than one order of magnitude. Results highlight that the presence of water content may cross the boundary between two radon risk categories, and consequently, may change the radon risk category towards lower risk categories. 


\section{ACKNOWLEDGEMENTS}

This work was supported by the Spanish Government [grant number RTI2018-099052-BI00] and the University of Alicante [grant number GRE17-12]. Special thanks to Dr. Marcel G. Schaap for his assistance on the Rosetta3 use. 


\section{REFERENCES}

Barbosa, S.M., R.V. Donner and G. Steinitz. 2015. Radon applications in geosciences Progress \& perspectives. Eur. Phys. J.: Spec. Top. 224: 597-603.

Benavente, D. and C. Pla. 2018. Effect of pore structure and moisture content on gas diffusion and permeability in porous building stones. Mater Struct 51.

Bossew, P. 2014. Determination of radon prone areas by optimized binary classification. J. Environ. Radioact. 129:121-132.

Davis, R.O.E. and H.H. Bennett. 1927. Grouping of soils on the basis of mechanical analysis. Washington D.C. (USA).

Edwards, K.B. 1994. Air permeability from pneumatic tests in oxidized till, J. Environ. Eng., $120(2): 329-346$.

Elío, J., Crowley, Q., Scanlon, R., Hodgson, J., Long, S., 2017. Logistic regression model for detecting radon prone areas in Ireland. Sci. Total Environ. 599-600:1317-1329.

Elío, J., Q. Crowley, R. Scanlon, J. Hodgson and L. Zgaga. 2018. Estimation of residential radon exposure and definition of Radon Priority Areas based on expected lung cancer incidence. Environ. Int. 114: 69-76.

Font, L. 2009. On radon surveys: Design and data interpretation. Radiat. Meas. 44: 964968.

Friske, P.W.B., K.L. Ford, R.J. McNeil, A.G. Pronk, M.A. Parkhill and T.A. Goodwin. 2014. Soil Geochemical,mineralogical, Radon and Gamma Ray Spectrometric Data from the 2007 North American Soil Geochemical Landscapes Project in New Brunswick, Nova Scotia, and Prince Edward Island. Geological Survey of Canada.

IAEA,International Atomic Energy Agency. 2013. Measurement and calculation of radon releases from NORM residues, Technical Reports Series no. 474, IAEA, Vienna

IPCC. 2013. Climate Change 2013: The Physical Science Basis. Contribution of Working Group I to the Fifth Assessment Report of the Intergovernmental Panel on Climate Change. Cambridge University Press, Cambridge, United Kingdom and New York, NY, USA.

Jones, A.P. 1999. Indoor air quality and health. Atmos. Environ. 33: 4535-4564.

Jury, W.A. and R. Horton. 2004. Soil Physics. Wiley, Mexico.

López-Abente, G., O. Núñez, P. Fernández-Navarro, J.M. Barros-Dios, I. Martín-Méndez, A. Bel-Lan, et al. 2018. Residential radon and cancer mortality in Galicia, Spain. Sci. Total Environ. 610-611: 1125-1132.

Mohammadi, M.H. and M. Vanclooster. 2019. A Simple Device for Field and Laboratory Measurements of Soil Air Permeability. Soil Science Society of America Journal.

Mualem, Y. 1976. A new model for predicting the hydraulic conductivity of unsaturated porous media. Water Resour. Res. 12: 513-522.

Nazaroff, W.W., 1992. Radon transport from soil to air. Rev. Geophys. 30: 137-160.

Nemes, A., M.G. Schaap, F.J. Leij and J.H.M. Wösten. 2001. Description of the unsaturated soil hydraulic database UNSODA version 2.0. J. Hydrol. 251: 151-162.

Neznal, M., M. Neznal, M. Matolín, I. Barnet and J. Mikšová. 2004. The new method for assessing the radon risk of building sites. Pr. Cesk. Geol. Ustavu: 7-47. 
Neznal, M., M. Neznal and J. Šmarda. 1995. Radon risk classification of foundation soils and other radon measurements by private firms. Bulletin Scientifique de l'Association des Ingénieurs Électriciens Sortis de l'Institut Montefiore 3: 31-34.

Pla, C., S. Cuezva, J. Martinez-Martinez, A. Fernandez-Cortes, E. Garcia-Anton, N. Fusi, et al. 2017. Role of soil pore structure in water infiltration and CO2 exchange between the atmosphere and underground air in the vadose zone: A combined laboratory and field approach. Catena 149: 402-416.

Pásztor, L., Szabó, K.Z., Szatmári, G., Laborczi, A., Horváth, Á., 2016. Mapping geogenic radon potential by regression kriging. Sci. Total Environ. 544:883-891.

Scanlon, B.R., J.P. Nicot and J.W. Massmann. 2001. Soil gas movement in unsaturated systems. In: A. W. Warrick, editor Soils Physics Companion. CRC Taylor \& Francis Group, Boca Raton FL (USA).

Schaap, M.G. and F.J. Leij. 1998. Database-related accuracy and uncertainty of pedotransfer functions. Soil Sci. 163: 765-779.

Schaap, M.G., F.J. Leij and M.T. Van Genuchten. 2001. Rosetta: A computer program for estimating soil hydraulic parameters with hierarchical pedotransfer functions. J. Hydrol. 251: 163-176.

Šimůnek, J., D. Jacques, G. Langergraber, S.A. Bradford, M. Šejna and M.T. Van Genuchten. 2013. Numerical modeling of contaminant transport using HYDRUS and its specialized modules. Journal of the Indian Institute of Science 93: 265-284.

Stauber, C.E., D. Dai, S.R. Chan, J.E. Diem, S.R. Weaver and R. Rothenberg. 2017. A pilot study to examine exposure to residential radon in under-sampled census tracts of Dekalb County, Georgia, in 2015. Int. J. Environ. Res. Public Health 14.

Szabó, K.Z., Jordan, G., Horváth, Á., Szabó, C., 2014. Mapping the geogenic radon potential: methodology and spatial analysis for central Hungary. J. Environ. Radioact. 129:107120.

Twarakavi, N.K.C., J. Šimůnek and M.G. Schaap. 2009. Development of Pedotransfer Functions for Estimation of Soil Hydraulic Parameters using Support Vector Machines Soil Science Society of America Journal 73: 1443-1452.

US Environmental Protection Agency. 2003. EPA Assessment of Risks from Radon in Homes. EPA 402-R-03-003. United States Environmental Protection Agency, Washington, DC (USA).

van Genuchten, M.T. 1980. Closed-form equation for predicting the hydraulic conductivity of unsaturated soils. Soil Science Society of America Journal 44: 892-898.

Van Looy, K., J. Bouma, M. Herbst, J. Koestel, B. Minasny, U. Mishra, et al. 2017. Pedotransfer Functions in Earth System Science: Challenges and Perspectives. Rev. Geophys. 55: 1199-1256.

Vienneau, D., K. De Hoogh, D. Hauri, A.M. Vicedo-Cabrera, C. Schindler, A. Huss, et al. 2017. Effects of radon and UV exposure on skin cancer mortality in Switzerland. Environ. Health Perspect. 125.

W. H. O. 2009. Handbook on indoor radon - A public health perspective. WHO Press, Geneva.

Watson, R.J., M.A. Smethurst, G.V. Ganerød, I. Finne and A.L. Rudjord. 2017. The use of mapped geology as a predictor of radon potential in Norway. J. Environ. Radioact. 166: $341-354$. 
Weeks, E.P. 1978. Field determination of vertical permeability to air in the unsaturated zone, 41, in U.S. G.S. Prof. Paper, USGS.

Zhang, H., H.J.H. Franssen, X. Han, J.A. Vrugt and H. Vereecken. 2017. State and parameter estimation of two land surface models using the ensemble Kalman filter and the particle filter. Hydrol. Earth Syst. Sci. 21: 4927-4958.

Zhang, Y., and Schaap, M.G. 2017. Weighted recalibration of the Rosetta pedotransfer model with improved estimates of hydraulic parameter distributions and summary statistics (Rosetta3). J. Hydrol. 547: 39-53.

Zhuo, W., Iida, T., Furukawa, M. 2006. Modeling radon flux density from the Earth's Surface, J. Nucl. Sci. Technol. 43: 479-482. 


\section{CAPTIONS}

Fig. 1. Relative permeability of gas and water versus water effective saturation for sand, silt and clay soil types. Hydraulic properties for each soil have been estimated using Rosetta3.

Fig. 2. Influence of bulk density, $\rho_{b}$, on the calculation of (a) intrinsic gas permeability expressed as $\mathrm{pK}_{\mathrm{g}}$ and (b) the parameter $n$ of the van Genuchten-Mualem model for sand, silt and clay soils using Rosetta3. Solid line considers soil texture (SSC) whereas dashed line includes both soil texture and bulk density (SSC+BD).

Fig. 3. $\mathrm{pK}_{\mathrm{g}}$ distribution in the USDA soil texture diagram for effective water saturations (a) $S_{w}=0.00$, (b) $S_{w}=0.20$, (c) $S_{w}=0.50$ and (d) $S_{w}=0.90$.

Fig 4. $\mathrm{pK}_{\mathrm{g}}$ versus effective water saturation, $\mathrm{S}_{\mathrm{w}}$, for sand, silt and clay soils. (a) General values and (b) zoom for lower $\mathrm{pK}_{\mathrm{g}}$.

Fig 5. Calculated unsaturated gas, $p K_{g}\left(S_{w}\right)$, and field gas, $p K_{g}$ field, permeabilities from the database of the Canadian component of NASGLP (Friske et al., 2014).

Fig 6. Potential radon risk based on Neznal et al., (1995) methodology. (a) Calculated gas permeability for different soil textures and water content $(\mathrm{Sw}=0,0.1,0.2$ and 0.4) for three values of radon gas concentration. (b) Comparison of the calculated intrinsic and unsaturated gas permeability with field radon gas concentration and permeability from the database of the Canadian component of NASGLP (Friske et al., 2014). 


\section{HIGHLIGHTS}

- Radon risk classification depends on the soil water content during the measurement

- Rosetta PTF estimate soil gas permeability from soil texture and water content

- The proposed methodology provides reliable gas permeability compared to field values 


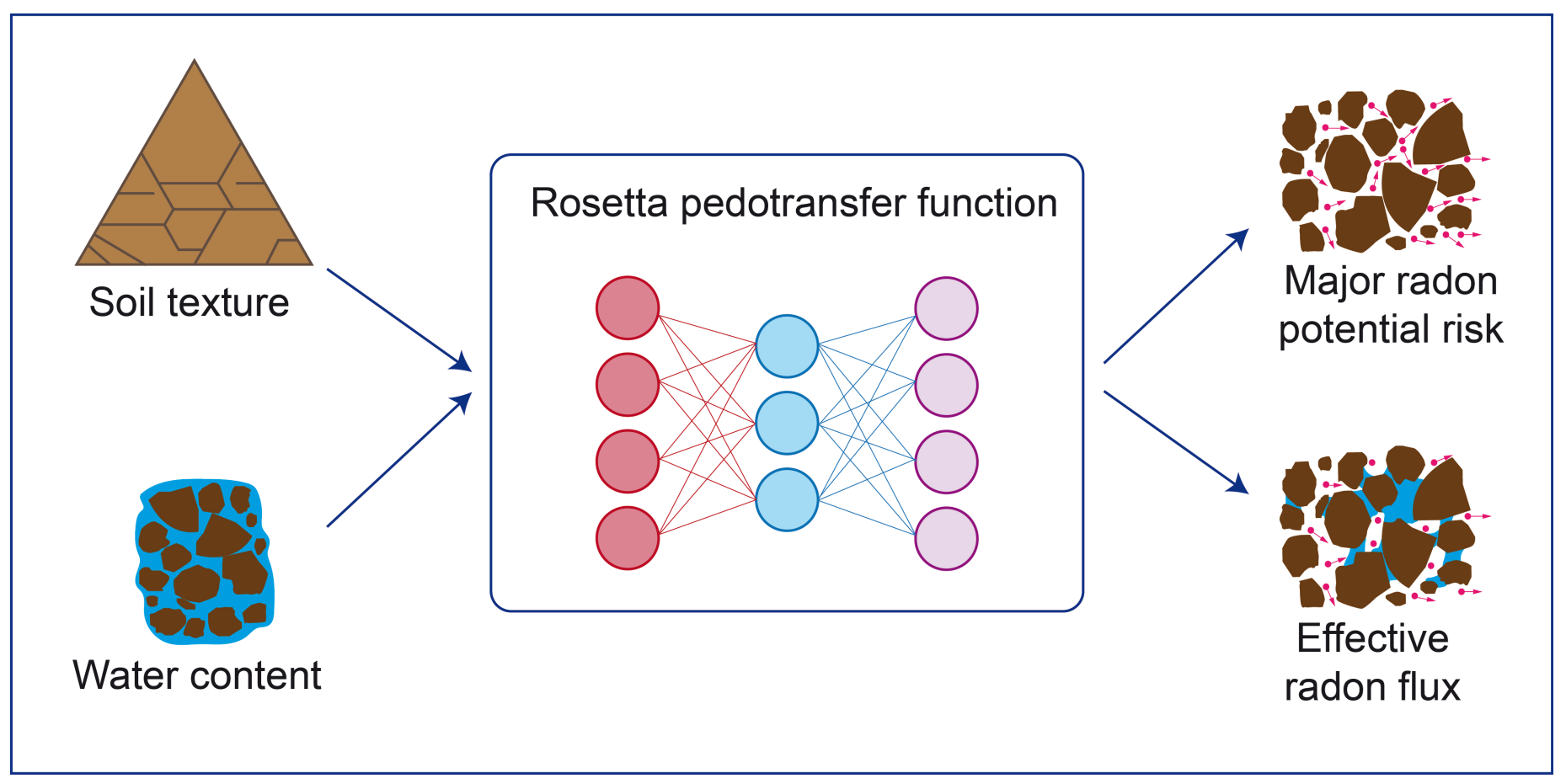




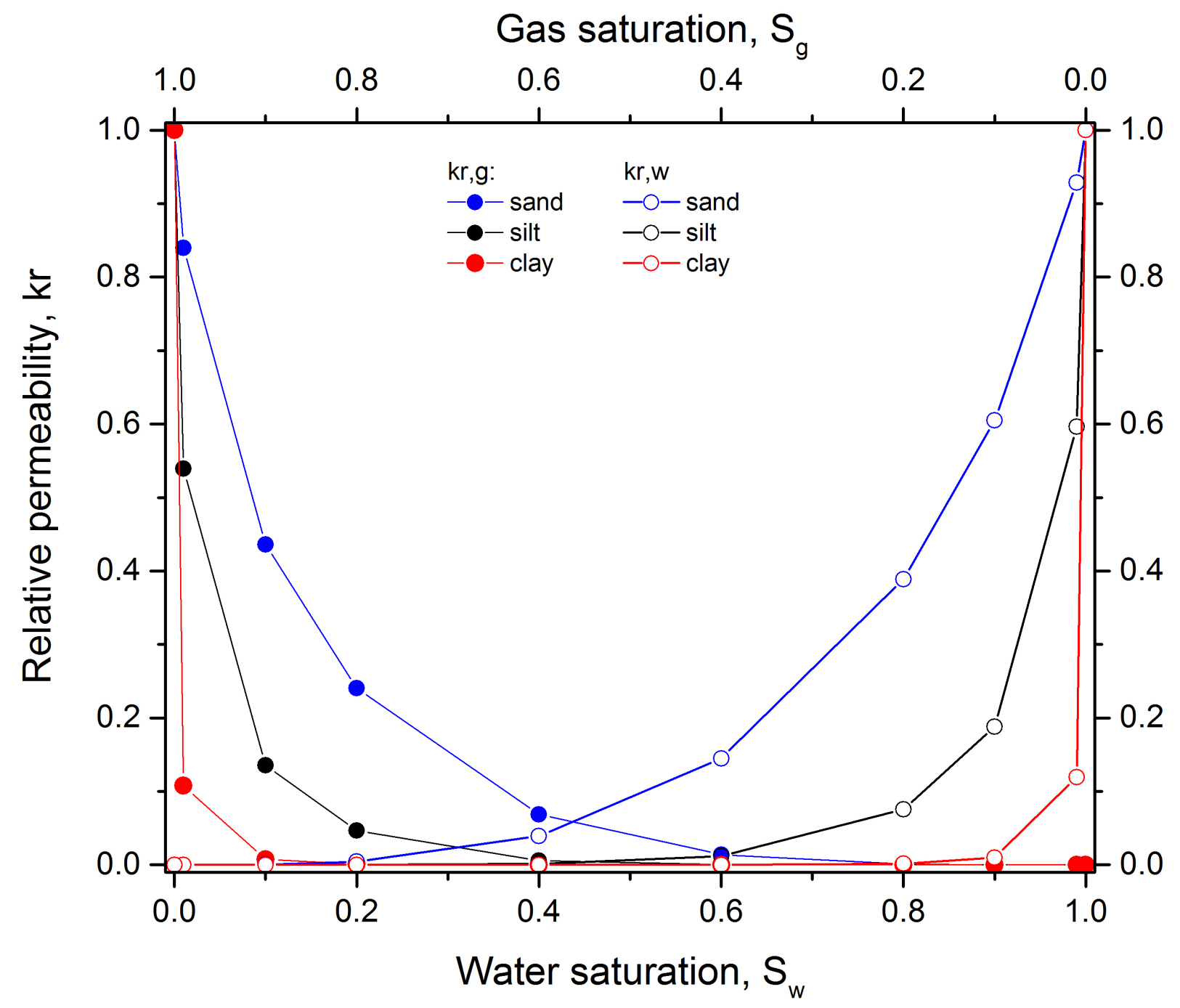


(a)

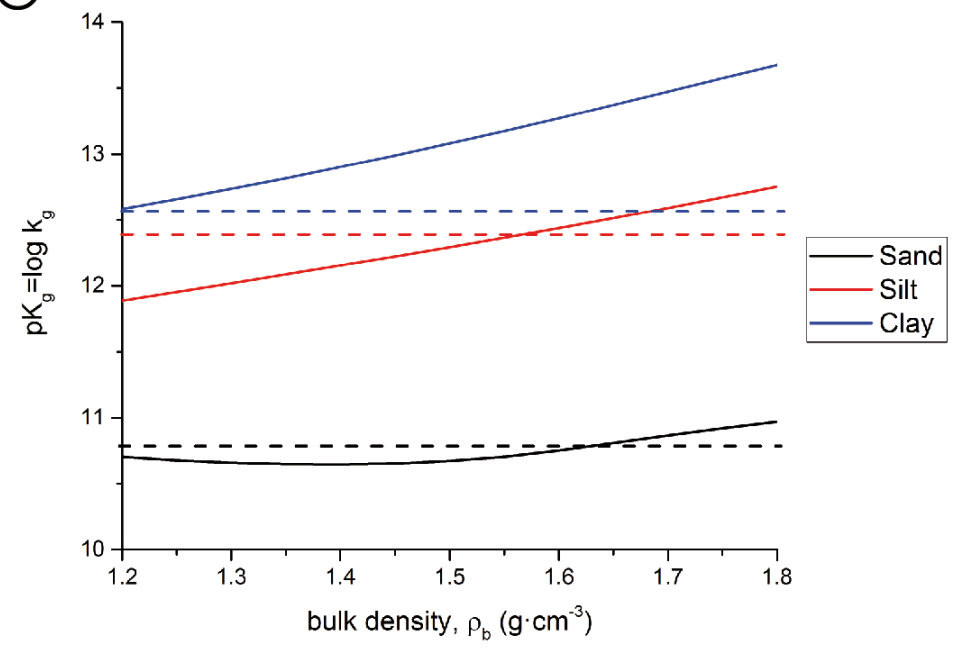

(b)

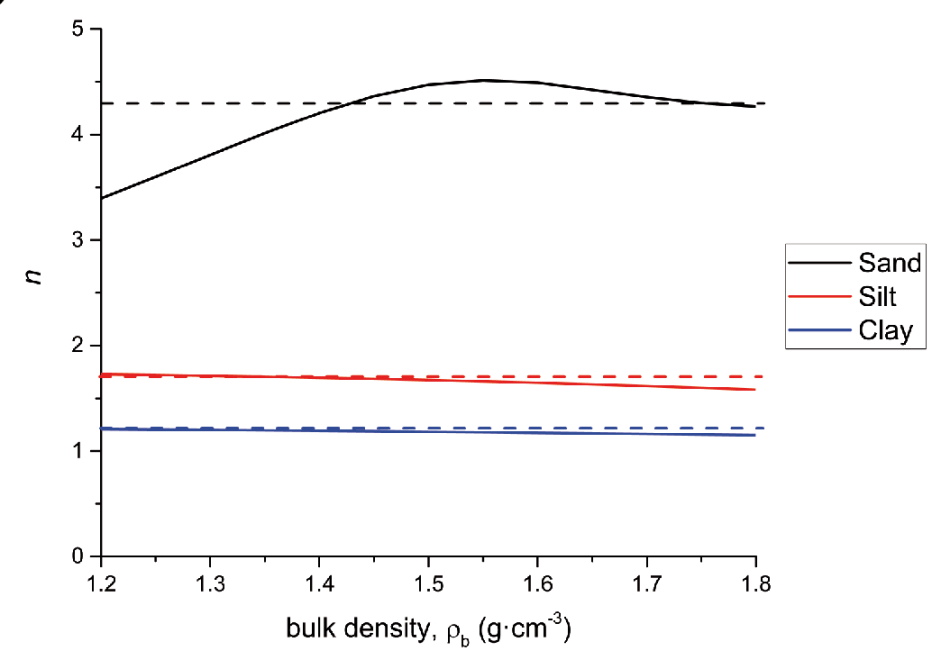



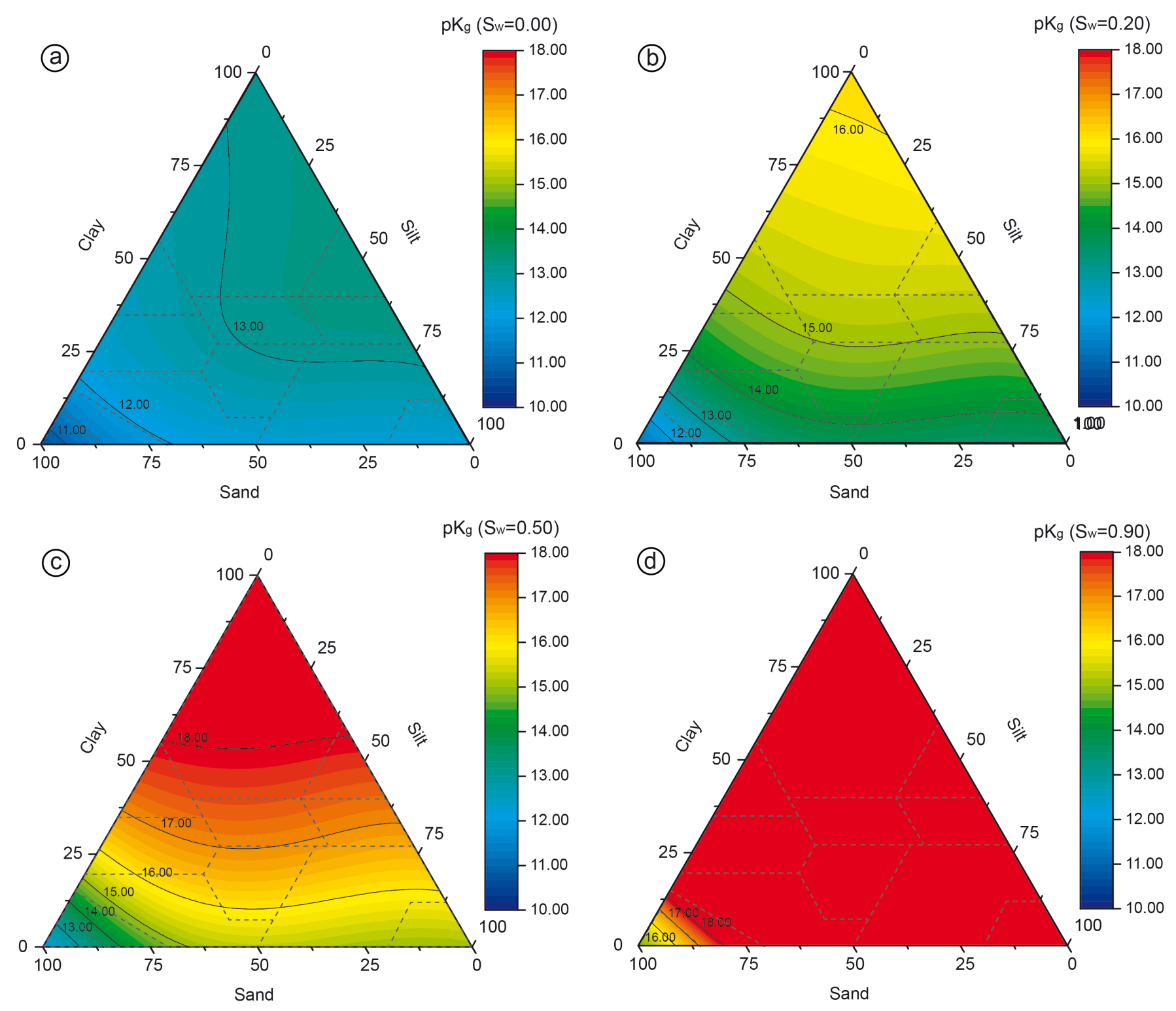
a)

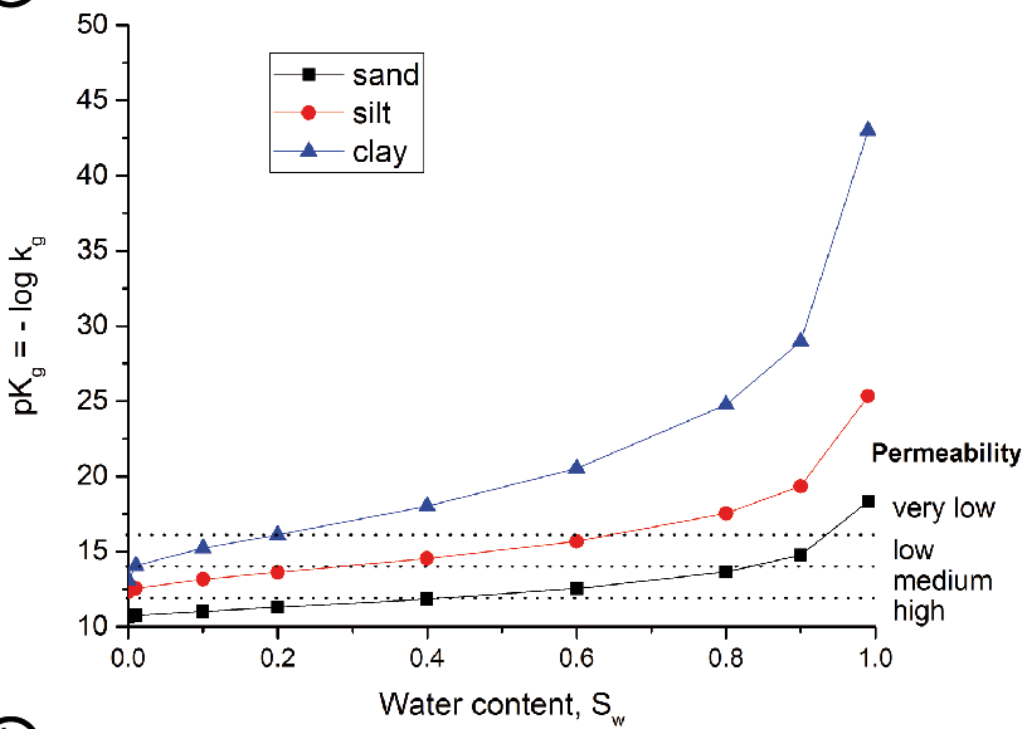

(b)

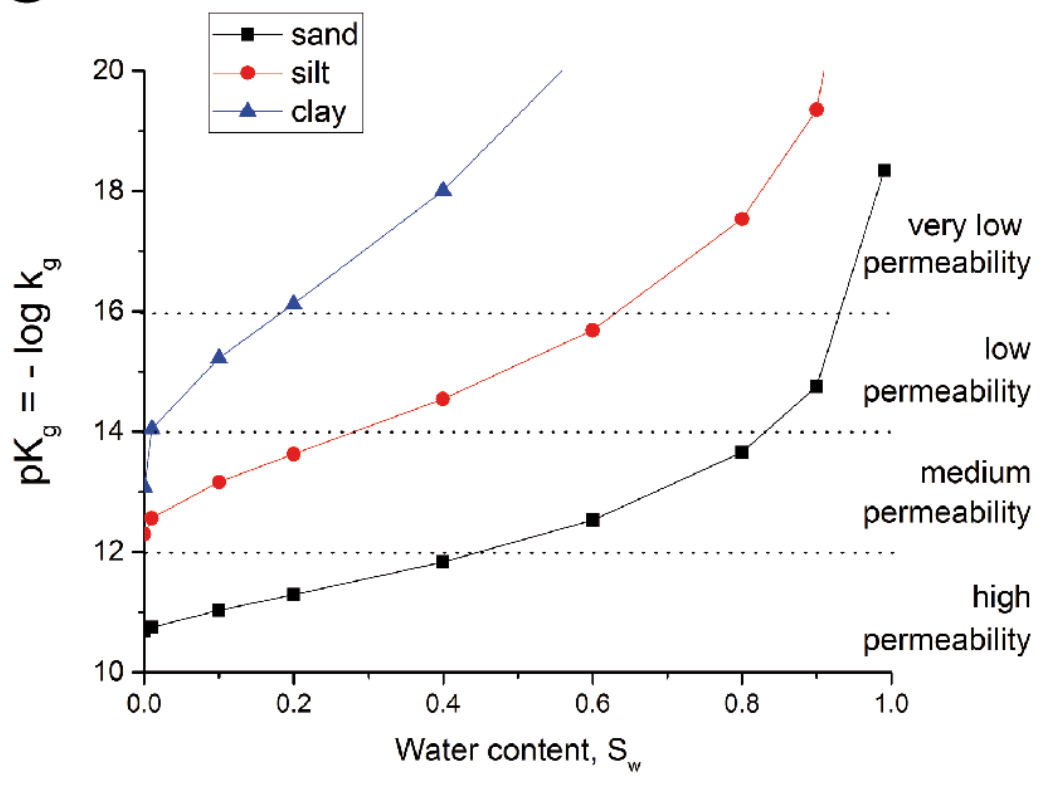




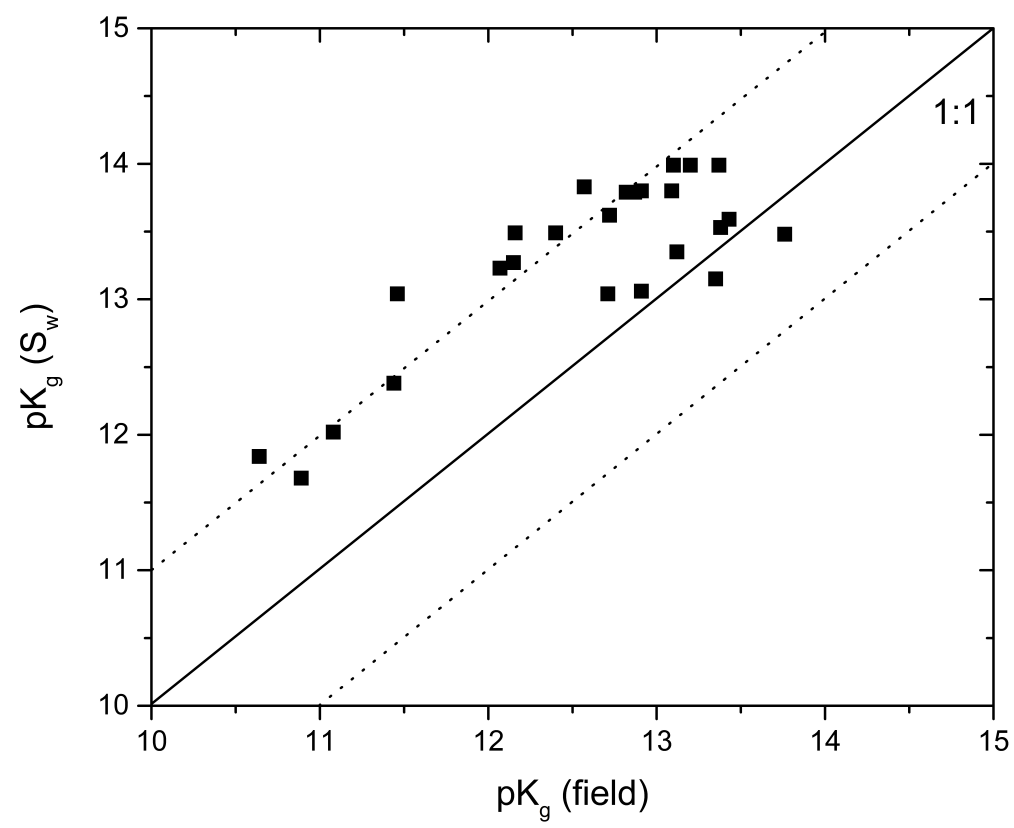


(a)

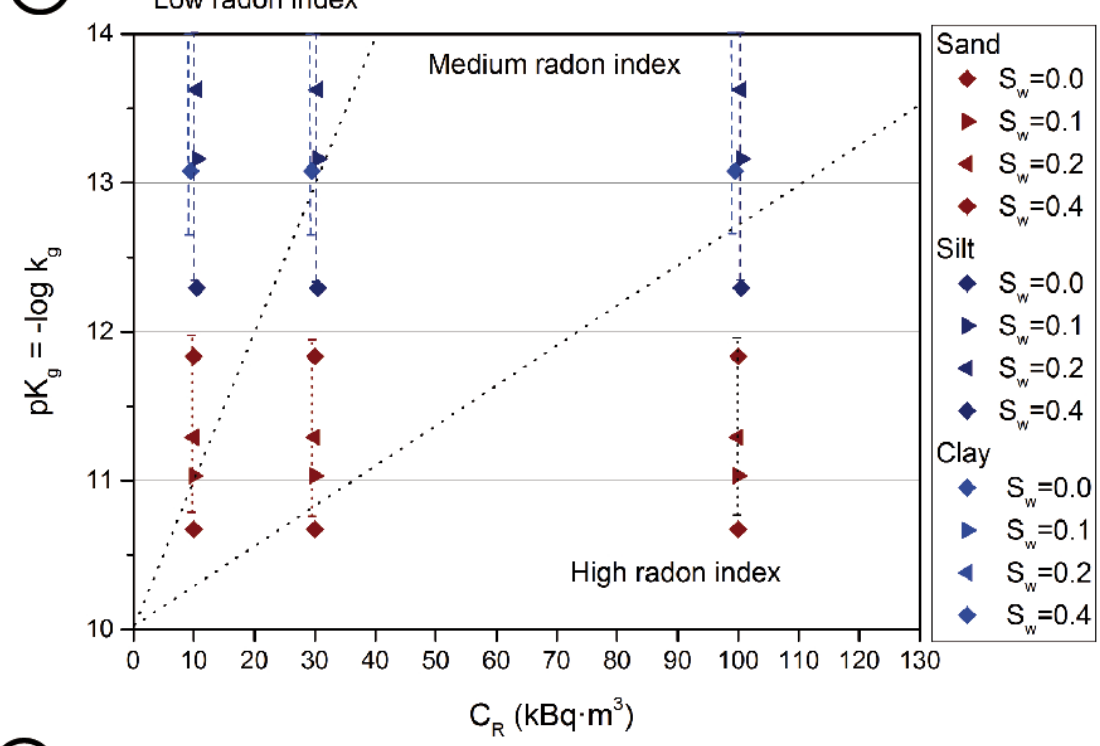

(b)

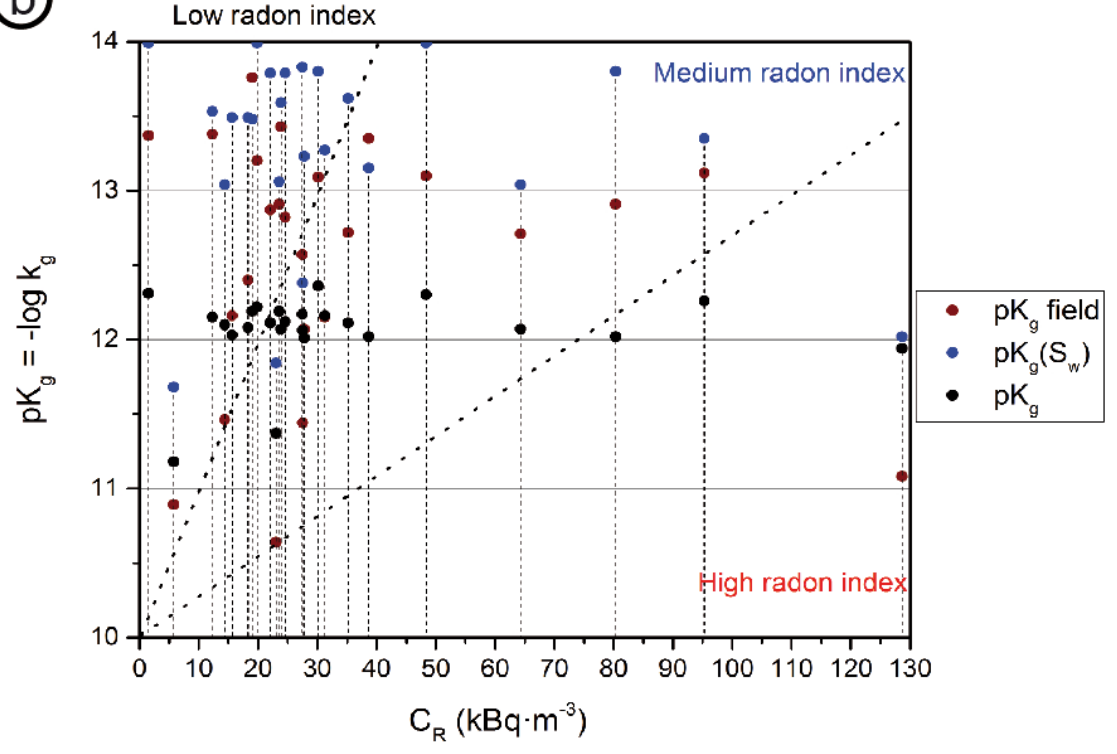




\section{Supplementary material}

(a)

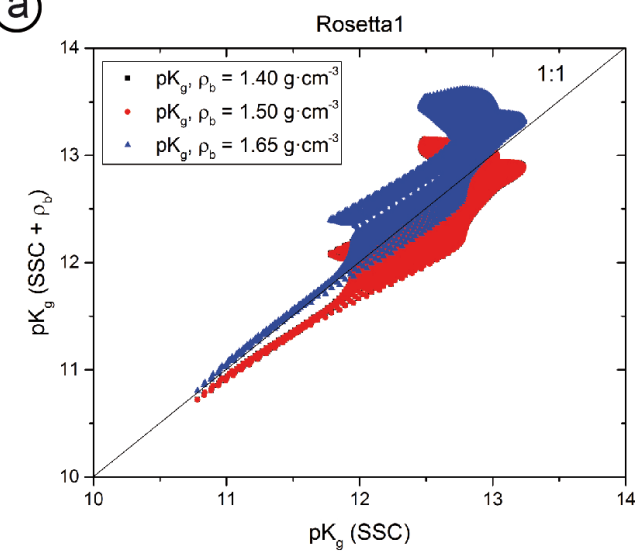

(C)



(b)

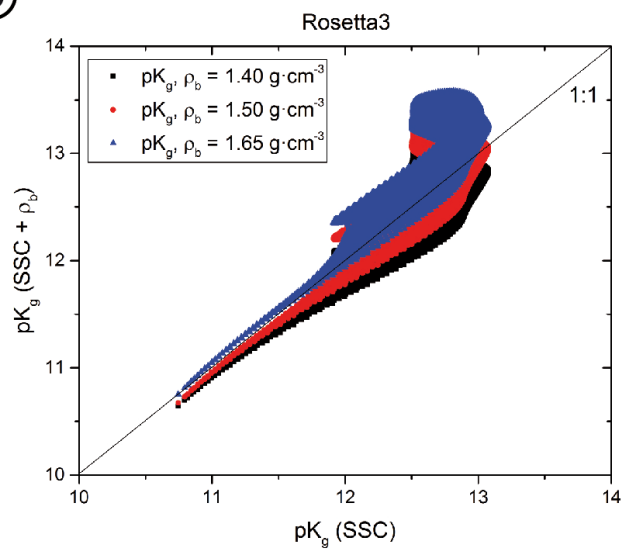

(d)

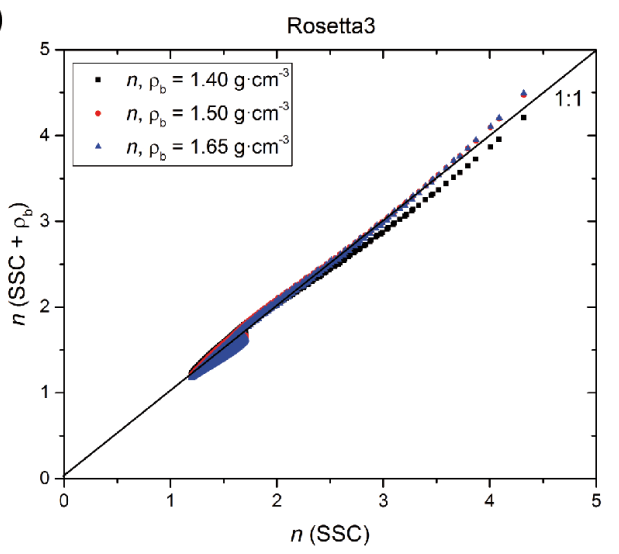

Figure S1. Influence of bulk density, $\rho_{b}$, on the calculation of (a) intrinsic gas permeability expressed as $\mathrm{pK}_{\mathrm{g}}$ and (b) the parameter $n$ of the van Genuchten-Mualem model using Rosetta1 and Rosetta3. $\mathrm{pK}_{\mathrm{g}}$ and $n$ estimations compare soil texture (SSC) and soil texture and for three bulk density values $\left(\mathrm{SSC}+\rho_{\mathrm{b}}\right)$. 
(a)

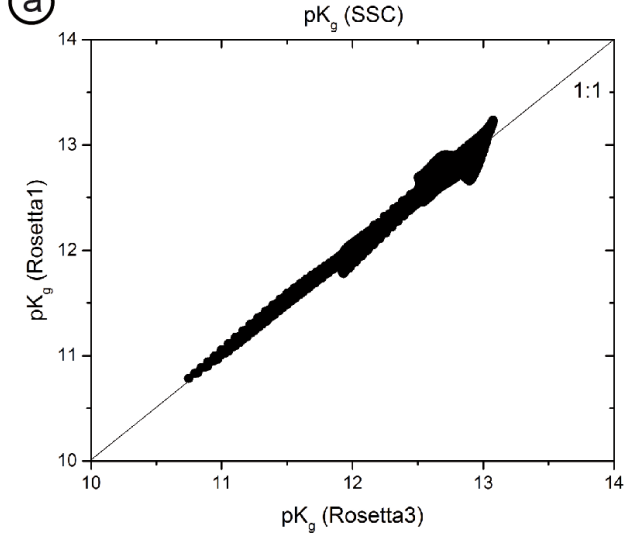

(C)

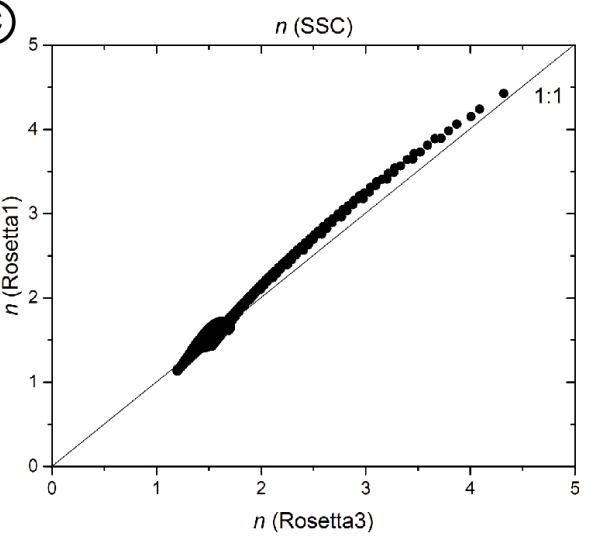

(b)

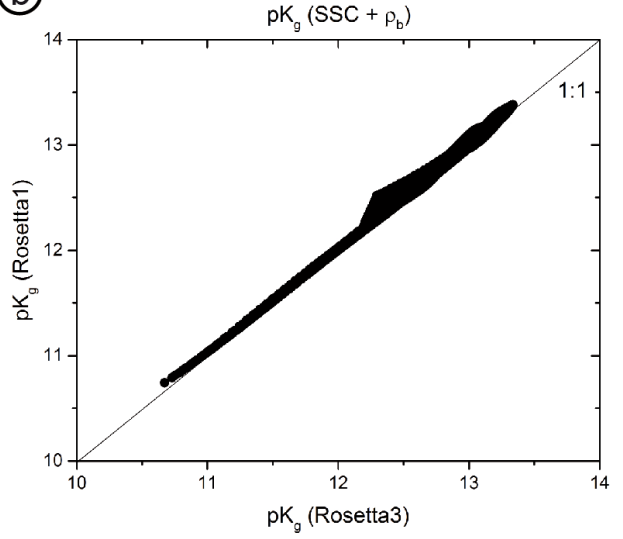

(d)

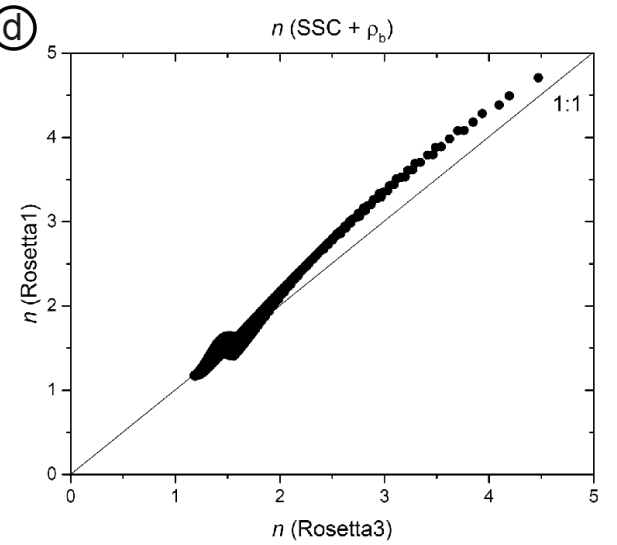

Figure S2. Estimation $\mathrm{pK}_{\mathrm{g}}$ and the parameter $n$ of the van Genuchten-Mualem model using Rosetta1 and Rosetta 3 and their comparison considering soil texture (SSC) and soil texture and a bulk density of $1.5 \mathrm{~g} \cdot \mathrm{cm}^{-3}\left(\mathrm{SSC}+\rho_{\mathrm{b}}\right)$. 
Table S1. $\mathrm{pK}_{\mathrm{g}}$ values for sand, silt and clay soils using Rosetta1 and Rosetta3 considering soil texture (SSC) and soil texture and for three bulk density values ( $\mathrm{pK}_{\mathrm{g}}=\log \mathrm{kg}_{\mathrm{g}}, \mathrm{kg}_{\mathrm{g}}$ is intrinsic gas permeability).

\begin{tabular}{|l|l|l|l|l|l|l|}
\hline \multirow{2}{*}{ Soil Texture } & \multicolumn{2}{|c|}{ Sand } & \multicolumn{2}{c|}{ Silt } & \multicolumn{2}{c|}{ Clay } \\
\cline { 2 - 7 } & Rosetta 1 & Rosetta 3 & Rosetta 1 & Rosetta 3 & Rosetta 1 & Rosetta 3 \\
\hline SSC & 10.782 & 10.746 & 12.357 & 12.381 & 12.671 & 12.543 \\
\hline SSC+1.40 $\cdot \cdot \mathrm{cm}^{-3}$ & 10.723 & 10.645 & 12.372 & 12.156 & 12.982 & 12.902 \\
\hline SSC+1.50 $\cdot \mathrm{cm}^{-3}$ & 10.742 & 10.673 & 12.519 & 12.294 & 13.116 & 13.081 \\
\hline SSC+1.60 $\cdot \mathrm{cm}^{-3}$ & 10.804 & 10.751 & 12.672 & 12.438 & 13.251 & 13.273 \\
\hline
\end{tabular}

Table S2. Values of the parameter $n$ of the van Genuchten-Mualem model for sand, silt and clay soils using Rosetta1 and Rosetta3 considering soil texture (SSC) and soil texture and for three bulk density values.

\begin{tabular}{|l|l|l|l|l|l|l|}
\hline Soil Texture & \multicolumn{2}{|c|}{ Sand } & \multicolumn{2}{c|}{ Silt } & \multicolumn{2}{c|}{ Clay } \\
\hline & Rosetta 1 & Rosetta 3 & Rosetta 1 & Rosetta 3 & Rosetta 1 & Rosetta 3 \\
\hline SSC & 4.425 & 4.319 & 1.615 & 1.693 & 1.155 & 1.200 \\
\hline SSC+1.40 $\cdot \mathrm{cm}^{-3}$ & 4.528 & 4.205 & 1.646 & 1.694 & 1.172 & 1.191 \\
\hline SSC+1.50 $\cdot \mathrm{cm}^{-3}$ & 4.705 & 4.472 & 1.636 & 1.672 & 1.175 & 1.182 \\
\hline SSC+1.60 $\cdot \mathrm{cm}^{-3}$ & 4.529 & 4.492 & 1.620 & 1.647 & 1.620 & 1.172 \\
\hline
\end{tabular}

